\title{
Alternatives to allogeneic blood transfusions
}

A

LTHOUGH the blood supply is safer today than it has ever been, public concern about the risks of hepatitis and HIV infections still exist. At the present time, the risk of contracting a HIV infection from a unit of red blood cells is reported to be 1 in 680,000 units in the United States and 1 in 913,000 in Canada. ${ }^{1,2}$ The approximate risk of developing hepatitis $B$ virus is 1 in 63,000 units and hepatitis $C$ is 1 in 100,000 in the United States. ${ }^{1}$ While these risks are much lower than the risk of death from a motor vehicle accident ( 1 in 9,594), the Krever report and publications in the popular press have alarmed patients throughout North America. ${ }^{2,3}$ In addition, the availability of allogeneic blood is not guaranteed. Elective surgery is occasionally canceled in Canadian cities because of a lack of available blood and a shortfall of four million units of red blood cells (RBCs) is projected in the United States by 2030 if the present rate of RBC utilization continues. ${ }^{4}$ Thus, every transfusion decision should include a risk/ benefit assessment; will the benefit of the transfusion to the patient outweigh the risk of complications and the cost of utilizing a precious commodity?

\section{Transfusion Trigger}

One alternative to a blood transfusion is "no transfusion" or a lowering of the "transfusion trigger." The old practice of transfusing a patient for a hemoglobin $(\mathrm{Hb})$ of less than $100 \mathrm{~g} \cdot \mathrm{l}^{-1}$ is no longer accepted. Current transfusion guidelines state that there is no clear and specific $\mathrm{Hb}$ level below which patients require transfusion. ${ }^{2,5}$ The decision to transfuse should be based on clinical parameters including symptoms, age, comorbidity, and vital signs in addition to the $\mathrm{Hb}$ level. Guidelines from both the Canadian Medical Association and the American Society of Anesthesiologists suggest that red cell transfusion is rarely indicated if the $\mathrm{Hb}$ level is $>100 \mathrm{~g} \cdot \mathrm{l}^{-1}$ and usually indicated if the $\mathrm{Hb}$ is $<60 \mathrm{~g} \cdot \mathrm{l}^{-1}$ in clinically stable patients not at risk for coronary artery disease.,

\section{Designated Blood Transfusion}

Designated blood transfusion, a practice in which the blood from a specific donor is reserved for a specific patient's use, is commonly requested by patients as an alternative to allogeneic blood transfusion. This transfusion practice is controversial because there is no evidence that designated blood is safer than allogeneic blood from volunteer donors and the risk of fatal graft $v s$ host disease exists. ${ }^{6,7}$ Moreover, this practice may have a deleterious effect of the recruitment of volunteer blood donors if people delay the donation of blood until a friend or family member has surgery.

\section{Preoperative Autologous Blood Donation}

Preoperative autologous blood donation (PAD) has become a standard of care in elective surgical procedures such as orthopedic and urological surgery. ${ }^{8,9}$ Guidelines for the selection of patients suitable for this technique and the utilization of this intervention have been published. ${ }^{10}$ Potential candidates for PAD before elective surgery include any patient scheduled for a procedure for whom blood type and cross-match is requested indicating a likelihood of requiring a blood transfusion according to a maximum surgical blood ordering schedule. Using this approach, studies have found that, overall, $9 \%$ of autologous blood donors undergoing elective surgery receive allogeneic blood. ${ }^{10}$ However, PAD has several limitations including high wastage rates of approximately 40 to $50 \%$ and poor cost effectiveness. ${ }^{11,12}$ Despite the fact that the blood given during PAD is the patient's own blood, adverse reactions to autologous blood do occur. ${ }^{13}$ Guidelines for the transfusion of PAD blood are controversial. Some published guidelines recommend that the same criteria be used for the transfusion of PAD blood as for allogeneic blood units. This view is supported by the risks of transfusion reactions and bacterial contamination. ${ }^{13,14}$ The alternative view holds that the risk of PAD blood is lower than for allogeneic blood since the infectious disease risk has been reduced. Despite the fact that controversy over this issue continues, physicians should not transfuse PAD blood simply because it is available.

\section{Acute Normovolemic Hemodilution}

Acute normovolemic hemodilution (ANH) is a technique that involves the removal of blood from a patient

From the Department of Anesthesiology, University of Florida College of Medicine, Gainesville, Florida 32610 USA. 
shortly before surgical blood loss, while restoring the circulating blood volume with acellular fluid. ${ }^{15}$ Blood is collected in standard blood bags containing anticoagulant and stored in the operating room at room temperature to preserve platelet, clotting factor, and white blood cell function. This blood is returned to the patient after major blood loss has ceased or sooner if indicated. The rationale for the use of hemodilution is that, if intraoperative blood loss is relatively constant with or without $\mathrm{ANH}$, it is better to lose blood at a lower rather than a higher hematocrit level.

Several studies have concluded that ANH is a costeffective alternative to $\mathrm{PAD}$ for patients undergoing elective surgery. ${ }^{15-17}$ Hemodilution as a "point of care" strategy in the operating room obviates the need for blood testing and eliminates costly blood wastage because all blood collected during ANH is returned to the patient. ${ }^{17}$ The risks of clerical error, transfusion reaction, and bacterial infection that exist with $\mathrm{PAD}$ or allogeneic blood are reduced or eliminated. Other advantages of ANH include patient convenience, ability to schedule surgery without delay, and the provision of fresh whole autologous blood for transfusion on the day of surgery.

\section{Preoperative Erythropoietin Therapy}

Erythropoietin, a glycoprotein released by the kidney in response to anemia and hypoxia, stimulates the bone marrow to increase red cell mass. Recombinant erythropoietin (EPO) is approved in the United States and Canada to increase the RBC mass of patients before elective surgery. ${ }^{18}$ Preoperative EPO therapy has been utilized to prevent anemia and increase the number of blood units collected during PAD. ${ }^{19} \mathrm{~A}$ recent study combined preoperative EPO therapy with intraoperative ANH in patients undergoing radical prostatectomy surgery. ${ }^{18}$ This study demonstrated that preoperative EPO was effective in minimizing the perioperative anemia associated with ANH and surgical blood loss and the transfusion of allogeneic blood compared to patients undergoing either $\mathrm{ANH}$ alone or PAD for blood conservation.

\section{Intraoperative Blood Salvage}

The term intraoperative blood salvage describes the technique of salvaging and reinfusing blood lost during surgery. The oxygen-transport properties of salvaged $\mathrm{RBCs}$ are equal to or better than stored allogeneic red cells and the survival of the salvaged RBCs appears to be at least comparable to that of allogeneic blood. ${ }^{20}$ Although the incidence of adverse events resulting from the reinfusion of salvaged blood is not known, it is thought to be an infrequent ( $<1: 15,000$ units) occur- rence. Problems associated with intraoperative blood salvage include the hemolysis of salvaged blood during suctioning of shed blood and dilutional coagulopathy if large volumes of salvaged blood are administered. The cost-effectiveness of intraoperative blood salvage has also been questioned. ${ }^{21,22}$ It is generally agreed that at least two blood unit equivalents need to be salvaged by the cell saver (with washed blood) in order to achieve cost-effectiveness. ${ }^{23}$

\section{Blood Substitutes}

Concerns over the safety and adequacy of the blood supply have increased interest in the development of a blood substitute. The term blood substitute is a misnomer because the products being developed as blood substitutes are actually oxygen-carrying volume expanders and are classified as drugs by the FDA. At the present time, the two types of oxygen-carrying fluids being developed are hemoglobin $(\mathrm{Hb})$ solutions and perfluorochemical (PFC) emulsions. ${ }^{4,24}$

In the early development of $\mathrm{Hb}$ solutions, problems with renal damage and intravascular coagulation were noted. Although the newer manufacturing techniques have eliminated many of the toxic side effects, all of the $\mathrm{Hb}$ solutions still appear to possess a vasoconstrictive effect which may be related to the binding of nitric oxide by the free hemoglobin solutions. ${ }^{4,24}$ Concerns about endotoxin contamination and immunogenicity also exist with several of the $\mathrm{Hb}$ solutions.

In the 1970s, the Green Cross Corporation in Japan developed Fluosol DA® 20\%, a PFC emulsion. At present, this is the only oxygen-carrying solution approved for clinical use and its use is restricted to the perfusion of coronary arteries after percutaneous transluminal coronary angioplasty. ${ }^{4,24}$ Problems with Fluosol included short intravascular half-life, low oxygen-carrying capacity, poor shelf life and temperature instability. Side effects associated with this product included an acute complement mediated reaction in some patients, marked uptake by the RES, and disruption of the normal pulmonary surfactant mechanism. ${ }^{25}$ Presently a second-generation PFC is under development. This newer emulsion is stable at room temperature and has a much higher oxygen-carrying capacity than Fluosol. While the safety profile of the newer PFC emulsion is much improved, limitations of this product include a short intravascular half-life of less than one day and the necessity for high inspired oxygen during administration in order for the drug to be effective.

A wide variety of potential clinical applications are possible with oxygen-carrying solutions. ${ }^{4,24}$ All of these solutions have low viscosities which give them rheolog- 
ic characteristics that may be helpful in the perfusion of ischemic organs. Both $\mathrm{Hb}$ and PFC solutions may be useful as resuscitative fluids prior to hospital admission and in combination with autologous blood techniques for intraoperative blood conservation. Other potential uses for these products include organ preservation, cardioplegic solutions, and enhancement of radiotherapy during cancer treatment. PFC solutions may also be developed as contrast dyes for CT or MRI procedures and therapy for carbon monoxide poisoning, air embolism, or decompression sickness.

\section{Conclusion}

The procurement of autologous blood is an accepted standard of care for elective surgery. However, the costs and potential complications, as well as the potential benefits of autologous blood procurement strategies, need to be reconsidered. In the future, blood conservation programs should combine cost-effective blood procurement strategies, such as ANH, with interventions that stimulate preoperative erythropoiesis. When released for clinical use, the addition of oxygen-carrying solutions to these blood conservation techniques will, hopefully, eliminate allogeneic blood use.

\section{References}

1 AuBuchon JP, Birkmeyer JD, Busch MP. Safety of the blood supply in the United States: Opportunities and controversies. Ann Intern Med 1997; 127: 904-9.

2 The Canadian Medical Association Expert Working Group. Guidelines for red blood cell and plasma transfusion for adults and children. Can Med Assoc J 1997; 156(11 Suppl): S1-24.

3 Gray C. Long before Krever's report, blood scare had changed face of medicine. Can Med Assoc J 1998; 158: 89-91.

4 Dietz NM, Joyner MJ, Warner MA. Blood substitutes: fluids, drugs, or miracle solutions? Anesth Analg 1996; 82: 390-405.

5 The American Society of Anesthesiologists Task Force on Blood Component Therapy: Practice guidelines for blood component therapy. Anesthesiology 1996; 84: 732-47.

6 Cordell RR, Talon VA, Cigahn-Haskell C, et al. Experience with 11,916 designated donors. Transfusion 1989; 26: 484-6.

7 Thaler $M$, Sharniss $A$, Orgad $S$, et al. The role of blood from HLA-homozygous donors in fatal transfusionassociated graft-versus-host disease after open-heart surgery. N Engl J Med 1989; 321: 25-8.

8 Goodnough LT, Shafron D, Marcus RE. The impact of preoperative autologous blood donation on orthopaedic surgical practice. Vox Sang 1990; 59: 65-9.
9 Toy PTCY, Menozzi D, Strauss RG, Stebling LC, Kruskall $M, A h n D K$. Efficacy of preoperative donation of blood for autologous use in radical prostatectomy. Transfusion 1993; 33: 721-4.

10 National heart, lung, and blood instirute expert panel on the use of autologous blood. Transfusion alert: use of autologous blood. Transfusion 1995; 35: 703-11.

11 Etchason J, Petz L, Keeler E, et al. The cost effectiveness of preoperative autologous blood donations. $\mathrm{N}$ Engl J Med 1995; 332: 719-24.

12 Goodnough LT, Grishaber JE, Birkmeyer JD, Monk TG, Catalona WJ. Efficacy and cost-effectiveness of autologous blood predeposit in patients undergoing radical prostatectomy procedures. Urology 1994; 44: 226-31.

13 Domen RE. Adverse reactions associated with autologous blood transfusion: evaluation and incidence at a large academic hospital. Transfusion 1998; 38: 301-6.

14 Sazama K. Reports of 355 transfusion-associated deaths: 1976 through 1985. Transfusion 1990; 30: 583-90.

15 Monk TG, Goodnough LT, Brecher ME, et al. Acute normovolemic hemodilution can replace preoperative autologous blood donation as a standard of care for autologous blood precurement in radical prostatectomy. Anesth Analg 1997; 85: 953-8.

16 Rottman G, Ness PM. Acute normovolemic hemodilution is a legitimate alternative to allogeneic blood transfusion. Transfusion 1998; 38: 477-80.

17 Monk TG, Goodnough LT, Brecher ME, Colberg JW, Andriole GL, Catalona WJ. A prospective randomized comparison of three blood conservation strategies for radical prostatectomy. Anesthesiology 1999; (in press).

18 Goodnough LT, Monk TG, Andriole GL. Erythropoietin therapy. N Engl J Med 1997; 336: 933-8.

19 Price TH, Goodnough LT, Vogler WR, et al. The effect of recombinant human erythropoietin on the efficacy of autologous blood donation in patients with low hematocrits: a multicenter, randomized, double-blind, controlled trial. Transfusion 1996; 36: 29-36.

20 Goodnough LT, Monk TG, Brecher ME. Autologous blood procurement in the surgical setting: lessons learned in the last 10 years. Vox Sang 1996; 71: 133-41.

21 Bell K, Stott K, Sinclair CJ, Walker WS, Gillon J. A controlled trial of intraoperative autologous transfusion in cardiothoracic surgery measuring effect on transfusion requirements and clinical outcome. Transfusion Med 1992; 2: 195-300.

22 Solomon MD, Rutledge ML, Kane LE, Yawn DH. Cost comparison of intraoperative autologous versus homologous transfusion. Transfusion 1988; 28: 370-82.

23 Bovill DF, Moulton CW, Jackson WS, Jenson JK, Barcellos $R W$. The efficacy of intraoperative autologous transfusion in major orthopaedic surgery: a regression analysis. Orthopaedics 1986; 9: 1403-7. 
24 Scott MG, Kucik DF, Goodnough LT, Monk TG. Blood substitutes: evolution and future applications. Clin Chem 1997; 43: 1724-31.

25 Hubmayr RD, Rodarte JR. Acute and long-term effects of Fluosol-DA 20\% on respiratory system mechanics and diffusion capacity in dogs. J Crit Care 1988; 3: 232-9. 


\section{Remplacer les transfu- sions sanguines allogéniques}

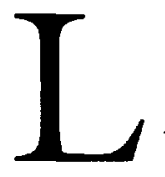
'APPROVISIONNEMENT en sang est plus sécuritaire aujourd'hui qu'il ne l'a jamais été, mais le public est toujours inquiet des risques d'infections aux virus VIH et de l'hépatite. Actuellement, le risque de contracter une infection au VIH à partir d'une unité de globules rouges est de 1 sur 680000 unités aux États-Unis et de 1 sur 913000 au Canada.,2 Dans le cas de l'hépatite $B$, le risque est d'environ 1 sur 63000 unités et pour l'hépatite C, de 1 sur 100000 aux États-Unis. ${ }^{1}$ Même si les risques sont plus bas que ceux de mourir dans un accident d'automobile (1 sur 9 594), le rapport Krever et les articles publiés dans la presse à grand tirage ont alarmé les patients de toute l'Amérique du Nord. ${ }^{2,3}$ De plus, la disponibilité du sang allogénique n'est pas assurée. Certaines opérations électives sont annulées au Canada par manque de sang, et on prévoit un déficit de quatre millions d'unités de globules rouges (GR) pour l'an 2030 aux États-Unis si leur utilisation se poursuit au rythme actuel. ${ }^{4}$ Par conséquent, toute décision de transfuser devrait s'accompagner d'une évaluation du rapport risque/bénéfices; est-ce que les bénéfices de la transfusion vont l'emporter sur le risque de complications et le coût d'utilisation d'un bien précieux?

\section{Le seuil de transfusion}

Une solution de rechange à la transfusion sanguine est «'absence de transfusion» ou l'abaissement du «seuil de transfusion». L'ancienne habitude de transfuser un patient lorsque le taux d'hémoglobine $(\mathrm{Hb})$ est de moins de $100 \mathrm{~g} \cdot \mathrm{l}^{-1}$ n'est plus acceptée. Les directives actuelles indiquent qu'il n'y a pas de niveau d'Hb spécifique sous lequel les patients ont besoin d'une transfusion. ${ }^{2,5} \mathrm{La}$ décision de transfuser devrait être basée sur des paramètres cliniques incluant les symptômes, l'âge, la comorbidité et les signes vitaux en plus du niveau d'Hb. Les directives de l'Association médicale canadienne autant que de l'American Society of Anesthesiologists (Société américaine des anesthésiologistes) suggèrent que la transfusion de GR est rarement indiquée si le niveau d'Hb est $>100 \mathrm{~g} \cdot \mathrm{l}^{-1}$ et est habituellement indiquée si l'Hb est $<60 \mathrm{~g} \cdot \mathrm{l}^{-1} \mathrm{chez}$ des patients dont l'état clinique est stable et qui ne sont pas à risque d'une maladie coronarienne:

\section{Transfusion sanguine désignée}

La transfusion sanguine désignée, une pratique dans laquelle le sang d'un donneur spécifique est réservé à l'usage d'un patient particulier, est régulièrement demandée par les patients pour remplacer la transfusion sanguine allogénique. Cette pratique est controversée, car rien ne prouve que le sang désigné soit plus sécuritaire que le sang de donneurs volontaires, et le risque d'une réaction fatale du greffon contre l'hôte, existe. ${ }^{6,7}$ Et qui plus est, cette pratique peut avoir un effet néfaste sur le recrutement de donneurs volontaires si les gens retardent leur don de sang jusqu'à ce qu'un ami ou un membre de leur famille soit opéré.

\section{Don de sang autologue préopératoire}

Le don de sang autologue préopératoire (DAP) est devenu une norme de soins lors des interventions chirurgicales électives, en orthopédie et en urologie par exemple. ${ }^{8,9}$ Des directives sur la sélection des patients, à qui la technique peut s'appliquer, et sur l'utilisation de cette intervention ont été publiées. ${ }^{10}$ Les candidats potentiels pour le DAP sont tous les patients pour qui l'opération est prévue et pour qui le groupe sanguin et l'épreuve de compatibilité croisée sont nécessaires, indiquant la probabilité d'un besoin de transfusion sanguine selon le schéma de perte maximale de sang chirurgical. Des études ont montré que, au total, selon cette méthode, $9 \%$ des donneurs de sang autologue qui subissent une opération élective ont reçu du sang allogénique. ${ }^{10}$ Cependant, le DAP présente certaines limites comme des pertes d'environ 40 à $50 \%$ et un faible rapport coût-efficacité. ${ }^{11,12}$ Malgré le fait que le sang donné pendant le DAP soit le propre sang du patient, des réactions indésirables peuvent survenir. ${ }^{13}$ Les directives concernant la transfusion de sang DAP sont controversées. Certaines recommandent que les mêmes critères s'appliquent aux transfusions de sang DAP qu'aux unités de sang allogénique. Cette vision est renforcée par les risques de réactions transfusionnelles et de contamination bactérienne. ${ }^{13,14} \mathrm{Par}$ ailleurs, on soutient que les risques de DAP sont plus 
faibles qu'avec le don de sang allogénique, puisque les risques de ma-ladies infectieuses ont été réduits. Malgré la controverse qui se poursuit, les médecins ne devraient pas transfuser du sang DAP parce qu'il est tout simplement disponible.

\section{Hémodilution normovolémique aiguë}

L'hémodilution normovolémique aiguë (HNA) consiste à prélever le sang du patient un peu avant la perte sanguine chirurgicale, tout en maintenant le volume de sang circulant avec un liquide acellulaire. ${ }^{15}$ Le sang recueilli dans des sacs standards contenant un anticoagulant, est gardé dans la salle d'opération à la température ambiante pour préserver les plaquettes, le facteur de coagulation et la fonction leucocytaire. Ce sang est retourné au patient après que la perte majeure de sang a cessé, ou plus tôt s'il y a lieu. La logique de l'utilisation de l'hémodilution c'est que, si la perte sanguine peropératoire est relativement constante avec ou sans HNA, il est préférable que cette perte se produise sous un niveau d'hématocrite plus faible que plus élevé.

Certaines études ont conclu que l'HNA est une solution de rechange rentable au DAP dans le cas d'une opération élective. ${ }^{15-17}$ L'hémodilution, faisant partie des soins de la salle d'opération, elle pare à la nécessité de tests sanguins et élimine un gaspillage coûteux parce que tout le sang collecté pendant l'HNA retourne au patient. ${ }^{17}$ Les risques d'erreur d'identification, de réaction transfusionnelle et d'infection bactérienne qui existent avec le DAP ou le sang allogénique sont réduits ou éliminés. Les autres avantages de l'HNA sont : l'aspect commode pour le patient, la possibilité de prévoir l'opération sans délai et l'approvisionnement de sang autologue frais et complet pour la transfusion le jour même de l'intervention.

\section{L'érythropoïttine préopératoire}

L'érythropoiétine, une glycoprotéine libérée par le rein en réaction à l'anémie et à l'hypoxie, stimule la moelle osseuse à produire des globules rouges. L'usage de l'érythropoïétine recombinante (EPO) est approuvée aux États-Unis et au Canada pour accroître la masse de GR des patients avant une opération élective. ${ }^{18}$ La thérapie EPO préopératoire a été utilisée pour prévenir l'anémie et augmenter le nombre d'unités de sang recueillies pendant le DAP. ${ }^{19}$ Une récente étude a combiné cette thérapie et l'HNA peropératoire pour des cas de prostatectomie radicale. ${ }^{18}$ L'étude a montré l'efficacité de l'EPO préopératoire pour diminuer l'anémie périopératoire associée à l'HNA et à la perte sanguine chirurgicale et à la transfusion de sang allogénique comparée à l'HNA seule ou au DAP pour la conservation du sang.

\section{Récupération de sang peropératoire}

La récupération de sang peropératoire est la technique de récupération et de reperfusion du sang perdu pendant l'opération. Les propriétés de transport d'oxygène des GR récupérés sont égales ou meilleures à celles des GR allogéniques conservés et la survie des GR récupérés est au moins comparable à celle du sang allogénique. ${ }^{20}$ Bien que l'incidence de complications résultant de la reperfusion de sang récupéré soit inconnue, on la croit faible ( $<1: 15000$ unités). Les problèmes associés à la récupération de sang comprennent l'hémolyse de sang récupéré pendant l'aspiration de sang perdu et la coagulopathie de dilution, si d'importants volumes de sang récupéré sont administrés. Le rapport coût-efficacité de la récupération de sang peropératoire a été aussi remise en question. ${ }^{21,22} \mathrm{On}$ s'entend généralement pour qu'au moins l'équivalent de deux unités de sang soit prélevé par le récupérateur (avec du sang lavé) pour atteindre une rentabilité satisfaisante. ${ }^{23}$

\section{Succédané du sang}

Les préoccupations entourant la sécurité et la fiabilité de l'approvisionnement de sang ont suscité l'intérêt pour l'élaboration d'un succédané du sang. Mais ce terme ne convient pas, parce que les produits qu'on a mis au point comme substitut du sang sont, en fait, des solutions de remplissage vasculaires transportant de l'oxygène et sont classés comme des médicaments par la FDA. Actuellement, les deux types existants de solutions transportant de l'oxygène sont des solutions d'hémoglobine $(\mathrm{Hb})$ et des émulsions chimiques perfluorées (CPF). 4,24

Au début de l'expérimentation de ces solutions, des problèmes de dommages rénaux et de coagulation intravasculaire ont été notés. Les nouvelles techniques de fabrication ont permis d'éliminer nombre d'effets secondaires, mais toutes les solutions d'Hb semblent posséder encore un effet vasoconstricteur qui peut être relié à la fixation de l'oxyde nitrique par les solutions d'hémoglobine libre.424 Des inquiétudes existent aussi au sujet de la contamination aux endotoxines et de l'immunogénicité avec certaines solutions d'Hb.

Dans les années 1970, la Green Cross Corporation au Japon a mis au point le Fluosol DA® 20\%, une émulsion CPF. Maintenant la seule solution transportant de l'oxygène qui soit approuvée pour l'usage clinique, elle est réservée à la perfusion des artères coronaires après une angioplastie coronaire transluminale percutanée. ${ }^{4,24}$ Les problèmes du Fluosol incluent une demi-vie intravasculaire brève, une faible capacité de transport d'oxygène, un temps limité de conservation et une instabilité à différentes températures. Les effets se- 
condaires en sont une réaction aiguë par médiation dụ complément chez certains patients, une accumulation marquée par le système réticulo-endothélial et l'altération du mécanisme normal du surfactant pulmonaire. ${ }^{25}$ Actuellement, on élabore une deuxième génération de $\mathrm{CPF}$ qui est stable à la température ambiante et qui a une beaucoup plus grande capacité de transport d'oxygène que le Fluosol. La sécurité de ce nouveau produit est nettement améliorée, malgré ses limites : demi-vie intravasculaire brève, de moins d'une journée, et nécessité d'une grande quantité d'oxygène inspiré pendant l'administration du médicament pour assurer l'efficacité de ce dernier.

De nombreuses applications cliniques potentielles sont possibles avec ces solutions transportant de l'oxygène. ${ }^{4,24}$ Toutes ces solutions ont une faible viscosité, ce qui leur donne des caractéristiques rhéologiques qui pourraient être utiles pour la perfusion d'organes ischémiques. Les deux solutions, $\mathrm{Hb}$ et $\mathrm{CPF}$, peuvent servir de liquides lors de la réanimation précédant l'admission à l'hôpital et peuvent être utilisées en combinaison avec des techniques de sang autologue pour la conservation du sang peropératoire. D'autres usages possibles incluent la préservation organique, les solutions cardioplégiques et la facilitation de la radiothérapie pendant le traitement du cancer. Les solutions CPF peuvent aussi servir comme substances de contraste pour la tomodensitométrie et l'IRM et dans la thérapie dans les cas d'intoxication au monoxyde de carbone, d'embolie gazeuse ou de mal de décompression.

\section{Conclusion}

L'acquisition de sang autologue fait partie des normes de traitement pour les interventions chirurgicales électives. Cependant, le coût et les complications potentielles, aussi bien que les bénéfices possibles des stratégies d'acquisition, doivent être réexaminés. Dorénavant, les programmes de conservation du sang devraient combiner des stratégies rentables d'acquisition, comme l'HNA, avec des interventions qui stimulent l'érythropoïèse préopératoire. Quand elles seront admises en pratique clinique, ces solutions transportant de l'oxygène, ajoutées aux techniques de conservation du sang, permettront, espérons-le, l'usage de sang allogénique.

\section{Références}

(Voir page $\mathrm{R} 5$ ) 